\title{
A novel, single-amplification PCR targeting mitochondrial genome highly sensitive and specific in diagnosing malaria among returned travellers in Bergen, Norway
}

${\text { Christel G Haanshuus }{ }^{1 *} \text {, Stein C Mohn¹, Kristine Mørch¹, Nina Langeland }{ }^{2} \text {, Bjørn Blomberg }}^{1,2}$ and Kurt Hanevik ${ }^{1,2}$

\begin{abstract}
Background: Nested PCR is a commonly used technique in diagnosis of malaria owing to its high sensitivity and specificity. However, it is time-consuming, open to considerable risk of contamination and has low cost-efficiency. Using amplification targets presented in multiple copies, such as rRNA 18S, or mitochondrial targets with an even higher copy number, might increase sensitivity.
\end{abstract}

Methods: The sensitivity and specificity of two newly designed Plasmodium genus-specific single-round amplification PCR programmes, based on previously published primers targeting 185 and mitochondrial genome, were compared with a widely used nested $18 \mathrm{~S}$ PCR. Analyses of dilution series from Plasmodium falciparum reference material were performed, as well as retrospective analyses of 135 blood samples, evaluated by routine microscopy, from 132 fever patients with potential imported malaria. Sequencing of the 220 bp mitochondrial PCR products was performed.

Results: At the threshold dilution 0.5 parasites/ $\mu$ l, the sensitivity of the mitochondrial PCR was 97\% (29/30 parallels), that of the single-round 18S PCR 93\% and the reference nested 18S PCR 87\%. All three assays detected as low as $0.05 \mathrm{p} / \mu \mathrm{l}$, though not consistently. In the patient cohort, malaria was diagnosed in 21\% (28/135) samples, defined as positive by at least two methods. Both single-round amplification assays identified all malaria positives diagnosed by nested PCR that had sensitivity of 96\% (27/28). The mitochondrial PCR detected one additional sample, also positive by microscopy, and was the only method with $100 \%$ sensitivity (28/28). The sensitivity and specificity of the mitochondrial PCR were statistically non-inferior to that of the reference nested PCR. Microscopy missed two infections detected by all PCR assays. Sequencing of the genus-specific mitochondrial PCR products revealed different single nucleotide polymorphisms which allowed species identification of the 28 sequences with following distribution; 20 P. falciparum, six Plasmodium vivax, one Plasmodium ovale and one Plasmodium malariae.

Conclusions: In this study, design of PCR programmes with suitable parameters and optimization resulted in simpler and faster single-round amplification assays. Both sensitivity and specificity of the novel mitochondrial PCR was $100 \%$ and proved non-inferior to that of the reference nested PCR. Sequencing of genus-specific mitochondrial PCR products could be used for species determination.

Keywords: Malaria, Diagnostics, PCR, Amplification, Sequencing, Mitochondrial DNA, 18S, Sensitivity, Gametocytes, Returned travellers

\footnotetext{
* Correspondence: christel.gill.haanshuus@helse-bergen.no

${ }^{1}$ National Centre for Tropical Infectious Diseases, Department of Medicine,

Haukeland University Hospital, Bergen, Norway

Full list of author information is available at the end of the article
} 


\section{Background}

More than three billion people world-wide are exposed to malaria, resulting in an estimated 200 million malaria cases and 1.2 million deaths in 2010 [1]. Routine diagnosis is usually done by microscopy and/or rapid diagnostic antigen detection tests, and may be influenced by factors such as the technologists' level of experience, and equipment quality [2,3]. Several molecular techniques have been developed, including polymerase chain reaction (PCR), with the aim of increasing the sensitivity and specificity $[4,5]$.

Malaria PCR was first introduced in 1990 [6], and subsequently has evolved with new amplification methods such as Real-Time PCR and loop-mediated isothermal amplification (LAMP) $[7,8]$. Both the latter methods have advantages over conventional PCR in turnaround time and in practical use as the detection step is incorporated into the amplification step. Nevertheless, the nested PCR originally described by Snounou et al. in 1993 [9] and improved in 1999 [10], remains commonly used and often regarded as a gold standard/reference method. Nested PCR exhibits high sensitivity and specificity due to two amplification steps, but is time-consuming, open to considerable risk of contamination and has low cost-efficiency [11-16].

High sensitivity has also been achieved by using amplification targets existing in multiple copies in the Plasmodium genome [17]. A common target is the conserved small subunit ribosomal RNA 18 S locus $[9,18,19]$ which in the Plasmodium falciparum chromosomal genome exists in five to eight copies depending on the strain [20]. Snounou et al. [9] reported a sensitivity of one to 10 parasites per microlitre $(\mathrm{p} / \mu \mathrm{l})$ of blood using nested PCR with $18 \mathrm{~S}$ as the target gene. Polley et al. [21] reported a sensitivity of $5 \mathrm{p} / \mu \mathrm{l}$ introducing a LAMP method using an amplification target on the $6 \mathrm{~kb}$ mitochondrial genome. In comparison, a LAMP assay employing primers targeting $18 \mathrm{~S}$ had a sensitivity limited to approximately 100 copies of the gene for P. falciparum [19]. Early ring stage $P$. falciparum parasites typically have one mitochondrial organelle, which contains about 20 copies of the $6 \mathrm{~kb}$ genome, while mature gametocytes have as much as four to eight mitochondrial organelles [22,23]. Although one would expect assays using mitochondrial targets to show higher sensitivity given the higher copy number, PCR-based methods targeting $18 \mathrm{~S}$ are commonly the methods of choice $[17,24]$.

The main aim of this study was to design practical single-round amplification Plasmodium genus-specific PCR assays, based on previously described primers targeting the 18S locus [9] and the mitochondrial genome [21], with sensitivity and specificity non-inferior to nested PCR [10]. Comparisons were performed using reference material and samples from a cohort of fever patients with potential imported malaria in Bergen, Norway.

\section{Methods}

Patient materials, positive controls and reference sample The patient material used in this study had been collected between 2006 and 2011 at Haukeland University Hospital, Bergen, Norway. It included 135 whole blood samples from a cohort of 132 fever patients with potential imported primary or recurrent malaria. As part of the routine work-up these samples had been previously analysed for malaria parasites on Giemsa-stained, thin and thick slides by experienced microscopists. The routine microscopy results and clinical information were collected retrospectively from patient files.

External DNA controls extracted from P. falciparum, Plasmodium vivax, Plasmodium ovale and Plasmodium malariae supplied by the Centre for Tropical Diseases, McGill University (Quebec, Canada) [25] were used in the validation of the genus-/ and species-specific PCR assays in the study. In addition, an external reference sample of P. falciparum, US 04 F Nigeria XII (World Health Organization, Geneva, Switzerland), was used to examine the sensitivity of the genus-specific PCR assays. $P$. falciparum was the only cultivated species accessible. The reference material contained exclusively ring stage parasites in a concentration of $200 \mathrm{p} / \mu \mathrm{l}$. Employing template from the reference material together with extracted DNA from blood of a Norwegian malaria negative volunteer, a combination of two 10-folds dilutions series were prepared giving the following series: $10 \mathrm{p} / \mu \mathrm{l}, 5 \mathrm{p} /$ $\mu \mathrm{l}, 1 \mathrm{p} / \mu \mathrm{l}, 0.5 \mathrm{p} / \mu \mathrm{l}, 0.1 \mathrm{p} / \mu \mathrm{l}, 0.05 \mathrm{p} / \mu \mathrm{l}$, and $0.001 \mathrm{p} / \mu \mathrm{l}$.

From all blood samples DNA was extracted using QIAamp DNA Blood Mini Kit (Qiagen, Hilden, Germany) according to the manufacturer's instructions. Both blood and extracted DNA material was stored at $-20^{\circ} \mathrm{C}$ prior to application.

\section{PCR methods}

Three PCR assays, two genus-specific and one speciesspecific, were assessed in the cohort of patient samples described above. The genus-/ and species-specific nested $18 \mathrm{~S}$ PCR as described by Singh et al. [10] was included as a reference method. In each PCR assay the reaction mixtures contained $2 \mu \mathrm{l}$ of DNA template and $12.5 \mu \mathrm{l}$ 2X HotStarTaq Master Mix (Qiagen) at a total volume of $25 \mu \mathrm{l}$. The amplifications were performed by using GeneAmp PCR System 9700 (Applied Biosystems, Carlsbad, CA, USA), and the PCR products were analysed by electrophoresis using $2 \%$ SeaKem ${ }^{\circledR}$ agarose gel (Lonza, Rockland, ME, USA) with $1 \mathrm{X}$ GelRed $^{\mathrm{TM}}$ (Biotium, Hayward, CA, USA). Concentrations of primers and additional $\mathrm{MgCl}_{2}$ were optimized for each assay examined as described below. 
One genus-specific PCR assay employed primers rPLU 6 and rPLU 5 (Table 1) targeting 18S. The amplification conditions were modified and optimized from the original nested [9] to a single-round amplification assay with cycle parameters as follows: step $1,95^{\circ} \mathrm{C}$ for $15 \mathrm{~min}$; step 2, denaturation at $95^{\circ} \mathrm{C}$ for $10 \mathrm{sec}$; step 3 , annealing at $63^{\circ} \mathrm{C}$ for $10 \mathrm{sec}$; step 4 , extension at $72^{\circ} \mathrm{C}$ for $75 \mathrm{sec}$; steps $2-4$ repeated 50 times; and step $5,72^{\circ} \mathrm{C}$ for $10 \mathrm{~min}$.

The other genus-specific assay was a new single-round amplification PCR using primers PgMt19 F3 and PgMt19 B3 (Table 1), targeting the mitochondrial genome and previously employed in a LAMP assay [21]. The primers were analysed using Oligo v6 primer analysis software (Molecular Biology Insights, Cascade, $\mathrm{CO}$, USA), and tested with Basic Local Alignment Search Tool (BLAST, National Center for Biotechnology Information, Bethesda, MD, USA) before being considered suitable for use in a conventional PCR assay. Subsequently, the following amplification conditions were designed: step $1,95^{\circ} \mathrm{C}$ for $15 \mathrm{~min}$; step 2, denaturation at $95^{\circ} \mathrm{C}$ for $10 \mathrm{sec}$; step 3 , annealing at $62^{\circ} \mathrm{C}$ for $10 \mathrm{sec}$; step 4 , extension at $72^{\circ} \mathrm{C}$ for $15 \mathrm{sec}$; steps $2-4$ repeated 50 times; and step $5,72^{\circ} \mathrm{C}$ for $10 \mathrm{~min}$. Reaction mixture for both assays contained $250 \mathrm{nM}$ of each primer, and additionally $4 \mathrm{mM} \mathrm{MgCl}_{2}$ (New England BioLabs, Ipswich, MA, USA).

The $P$. falciparum, $P$. vivax, $P$. ovale and $P$. malariae species-specific PCR protocol was employed on all genusspecific PCR positive samples applying primers targeting $18 \mathrm{~S}$ previously published by Padley et al. [26]. As opposed to the original multiplex assay, each sample was analyzed in four separate reaction mixtures to avoid difficulties in species interpretation due to similar product sizes. Only a few single nucleotide polymorphisms (SNPs) distinguish the different forward primers' hybridization sites. In order to avoid non-specific cross-binding between $P$. falciparum and $P$. vivax samples, a new $P$. vivax forward primer (Table 1) was designed using Oligo v6, the sequence alignment editor software BioEdit v7 (Tom Hall, Carlsbad, CA,
USA) and BLAST. The amplification conditions were modified and amplification time substantially reduced from six to two hours. The new cycling parameters were: step $1,95^{\circ} \mathrm{C}$ for $15 \mathrm{~min}$; step 2 , denaturation at $95^{\circ} \mathrm{C}$ for $10 \mathrm{sec}$; step 3 , annealing at $65^{\circ} \mathrm{C}$ for $10 \mathrm{sec}$; step 4, extension at $72^{\circ} \mathrm{C}$ for $30 \mathrm{sec}$; steps $2-4$ repeated 45 times; and step $5,72^{\circ} \mathrm{C}$ for $10 \mathrm{~min}$. The reaction mixtures contained additional $\mathrm{MgCl}_{2}$ which was optimized as follows: $4 \mathrm{mM}$ for P. ovale, $2 \mathrm{mM}$ for P. falciparum and P. malariae, and $1 \mathrm{mM}$ for $P$. vivax. The primer concentrations were 250 $\mathrm{nM}$ with an exception of $150 \mathrm{nM}$ for $P$. ovale.

\section{Sequencing}

For quality assurance purposes, all PCR products from genus-specific positive samples using primers PgMt19 F3\&B3 (Table 1) were sequenced in both directions. The PCR products were purified with ExoSAP-IT ${ }^{\circledR}$ (USB Corporation, Cleveland, $\mathrm{OH}$, USA) according to the manufacturer's instructions, prior to the following cycle conditions being applied using GeneAmp PCR System 9700 (Applied Biosystems): step $1,96^{\circ} \mathrm{C}$ for $10 \mathrm{sec}$; step $2,62^{\circ} \mathrm{C}$ for $5 \mathrm{sec}$; step $3,60^{\circ} \mathrm{C}$ for $4 \mathrm{~min}$; steps $1-3$ repeated 27 times. Each reaction mixture contained $1 \mu \mathrm{l}$ BigDye v1.1 (Applied Biosystems), $2 \mu \mathrm{l}$ sequencing buffer 5X (Applied Biosystems), $0.5 \mu \mathrm{M}$ primer, and $1 \mu \mathrm{l}$ template at a total volume of $10 \mu \mathrm{l}$. The sequences were obtained with the ABI PRISM ${ }^{\circledR} 3730$ DNA Analyzer (Applied Biosystems). BioEdit v7 was used prior to sequence identification using BLAST.

\section{Statistical methods}

Proportions were compared applying prtest command using Stata 11 (Stata Corp, College Station, Texas, USA), and differences in sensitivity and specificity with 95\% confidence intervals $(95 \% \mathrm{CI})$ were calculated. Noninferior of the test was considered proved if the upper boundary of the $95 \% \mathrm{CI}$ of the sensitivity difference was less than a predefined delta of $5 \%$.

Table 1 Primers applied for the amplifications and sequencing examined in this study

\begin{tabular}{lll}
\hline Primer $^{1}$ & Sequence & Published by \\
\hline rPLU 6 forward & $5^{\prime}$-tta aaa ttg cag tta aaa cg & Snounou et al. [9] \\
rPLU 5 reverse & $5^{\prime}$-cct gtt gtt gcc tta aac ttc & Snounou et al. [9] \\
PgMt19 F3 forward & $5^{\prime}$-tcg ctt cta acg gtg aac & Polley et al. [21] \\
PgMt19 B3 reverse & $5^{\prime}$-aat tga tag tat cag cta tcc ata g & Polley et al. [21] \\
Plasmodium falciparum forward & $5^{\prime}$-aac aga cgg gta gtc atg att gag & Padley et al. [26] \\
Plasmodium vivax forward & $5^{\prime}$-gag cgt tca aag caa aca ga & This study \\
Plasmodium ovale forward & $5^{\prime}$-ctg ttc ttt gca ttc ctt atg c & Padley et al. [26] \\
Plasmodium malariae forward & $5^{\prime}$-cgt taa gaa taa acg cca agc g & Padley et al. [26] \\
Species-specific reverse & $5^{\prime}$-gta tct gat cgt ctt cac tcc c & Padley et al. [26] \\
\hline
\end{tabular}

\footnotetext{
${ }^{1}$ The primers were obtained from Eurogentec (Seraing, Belgium).
} 


\section{Ethics}

The study was approved by the Regional Committee for Ethics in Medical Research (No.2011/942).

\section{Results}

The sensitivity of detection was examined for the three genus-specific amplification assays; the new single-round amplification mitochondrial PCR employing primers PgMt19 F3\&B3 [21], the modified single-round amplification 18S PCR employing primers rPLU 6\&5 [9], and nested 18S PCR [10] as the reference method. Based on 30 parallels of the described dilution series, the $0.5 \mathrm{p} / \mu \mathrm{l}$ dilution proved to be the threshold detection level for all three assays (Figure 1). The mitochondrial PCR detected $0.5 \mathrm{p} / \mu \mathrm{l}$ with $97 \%$ sensitivity $(29 / 30)$, while the modified $18 \mathrm{~S}$ PCR and nested $18 \mathrm{~S}$ PCR detected $0.5 \mathrm{p} / \mu \mathrm{l}$ with $93 \%(28 / 30)$ and $87 \%(26 / 30)$ sensitivity, respectively. At the threshold detection level of $0.5 \mathrm{p} / \mu \mathrm{l}$, the sensitivity of the mitochondrial PCR, was statistically non-inferior to that of the reference nested $18 \mathrm{~S}$ PCR, as the upper boundary of the $95 \%$ CI $(-23.8 \%$ to $3.8 \%)$ of the sensitivity difference $(10.0 \%)$ was less than the predefined delta of $5 \%$. Although, the sensitivity of the modified 18S PCR also was higher than that of the reference nested $18 \mathrm{~S}$ PCR in absolute numbers, statistical non-inferiority could not be proved in this sample size (sensitivity difference $-6.7 \%$, 95\% CI $-21.8 \%$ to $8.4 \%$ ). All three assays detected positive DNA as low as $0.05 \mathrm{p} / \mu \mathrm{l}$, though not consistently so by any method.

After having determined sensitivity of detection in standardized material, the 135 patient samples (132 patients) were screened for the presence of malaria by the three genus-specific PCR assays. Among these samples, $21 \%(28 / 135)$ were defined as malaria positive by at least two of the methods among microscopy and the three different genus-specific PCR assays. The new mitochondrial PCR was $100 \%$ sensitive detecting all 28 positives. Both the modified $18 \mathrm{~S}$ PCR and the nested $18 \mathrm{~S}$
PCR detected 27 of the positives corresponding to a sensitivity of $96 \%$. Routine microscopy detected 26 of the positives corresponding to a sensitivity of $93 \%$ (Table 2). The mitochondrial PCR detected one positive sample not detected by any of the two 18S PCR assays, which was also positive by microscopy. Two positive samples not detected by microscopy were detected by all three PCR assays (Table 2). The sensitivity of the mitochondrial PCR was statistically non-inferior to that of the reference $18 \mathrm{~S}$ PCR, as the upper boundary of the $95 \% \mathrm{CI}$ $(-10.4 \%$ to $3.3 \%)$ of the sensitivity difference $(-3.6 \%)$ was less than the predefined delta of $5 \%$. Although the sensitivity of the modified $18 \mathrm{~S}$ PCR was equal to that of the reference nested $18 \mathrm{~S}$ PCR in absolute numbers, statistical non-inferiority could not be proved in this patient cohort (sensitivity difference $0 \%, 95 \%$ CI $-9.7 \%$ to $9.7 \%$ ).

None of the malaria-negative patients had false positive tests by any of the diagnostic methods, thus all the tests had $100 \%$ specificity in this cohort. The specificity of both the mitochondrial PCR and the modified 18S PCR were statistically non-inferior to that of the reference nested 18S PCR (specificity difference 0.0\%, 95\% CI 0.0 to $0.0 \%$ ).

The 28 malaria positive samples were all further examined by two species-specific $18 \mathrm{~S}$ amplification assays; an assay modified from a multiplex PCR [26], and speciesspecific nested PCR assay [10] as the reference method. The first assay, which was modified from a multiplex into four separate reactions with a new $P$. vivax forward primer, as described above, showed no cross-bindings between the new $P$. vivax primer and $P$. falciparum positive samples (Figure 2). Previously observations showed that the original $P$. vivax forward primer unspecifically cross-bonded with $P$. falciparum positive samples, especially when the parasitaemia was high

Sequencing of the genus-specific mitochondrial PCR products was performed giving high-quality sequences of full length. When the sequences were run through

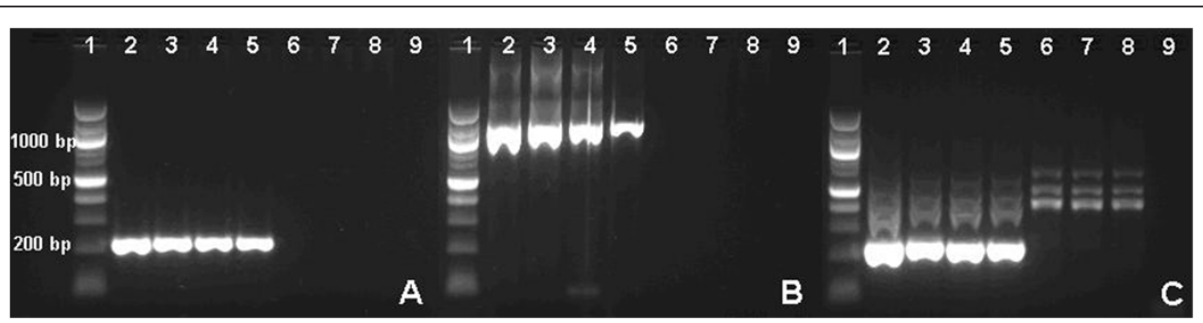

Figure 1 Sensitivity of three different Plasmodium genus PCR protocols. A dilution series, run in 30 parallels, was prepared from a $200 \mathrm{p} / \mu \mathrm{l}$ stock of Plasmodium falciparum, US 04 F Nigeria XII. The 2\% agarose gel picture shows a typical parallel for each assay; new single-round amplification mitochondrial PCR employing primers PgMt19 F3 \& B3 [21] (A), modified single-round amplification 18S PCR employing primers rPLU 6 \& 5 [9] (B), and gold standard genus-specific nested 18S PCR [10] (C). The product sizes are 220 base pair (bp), 1200 bp, and 250 bp, respectively. Lane $1=100 \mathrm{bp}$ DNA Ladder (New England BioLabs), lane $2=10 \mathrm{p} / \mu \mathrm{l}, 3=5 \mathrm{p} / \mu \mathrm{l}, 4=1 \mathrm{p} / \mu \mathrm{l}, 5=0.5 \mathrm{p} / \mu \mathrm{l}, 6=0.1 \mathrm{p} / \mu \mathrm{l}, 7=0.05 \mathrm{p} / \mu \mathrm{l}$, $8=0.001 \mathrm{p} / \mu \mathrm{l}$, and $9=$ no template. The new mitochondrial PCR had more defined bands than the modified $18 \mathrm{~S}$ PCR, and especially the nested $18 \mathrm{~S}$ PCR. 
Table 2 Genus-specific results from a cohort of 132 fever patients with potential imported malaria

\begin{tabular}{lllll}
\hline Samples $(n=135)$ & $\begin{array}{l}\text { New single-round amplification } \\
\text { mitochondrial (PCRPgMt19 F3 \& B3 [21]) }\end{array}$ & $\begin{array}{l}\text { Modified single-round amplification } \\
\text { 18S PCR (rPLU 6 \& 5 [9]) }\end{array}$ & $\begin{array}{l}\text { Genus-specific nested } \\
\text { 18S PCR [10] }\end{array}$ & $\begin{array}{l}\text { Microscopy } \\
\text { Positive }\end{array}$ \\
Negative & 28 & 27 & 27 & 26 \\
\hline
\end{tabular}

BLAST the results showed two to six SNPs and one insert/deletion which allowed species determination in all the 28 sequences, revealing 20 P. falciparum, six $P$. vivax, one $P$. ovale, and one $P$. malariae sequences (Figure 3, Table 3). Both species-specific 18S PCR assays identified $18 P$. falciparum, six $P$. vivax, one $P$. ovale, and one $P$. malariae single infections, and one double infection of $P$. falciparum and $P$. malariae (Table 3 ). While the routine microscopy had identified 20 P. falciparum, three P.m vivax, and one Plasmodium knowlesi/inconclusive single infections, and two samples with $P$. falciparum in double infection with $P$. ovale and $P$. vivax, respectively (Table 3).

In seven samples (six patients) the different methods had discordant results (Table 4). Based on the combined results from routine microscopy, all PCR assays and sequencing, these samples were evaluated giving the following interpretations; sequencing as a diagnostic method missed Plasmodium malariae in a double infection of $P$. falciparum and P. malariae, all 18S PCR assays (genus-/and species-specific) missed one $P$. falciparum positive sample detected by both the new mitochondrial PCR and microscopy, and routine microscopy missed one $P$. malariae and one $P$. vivax single infection as well as

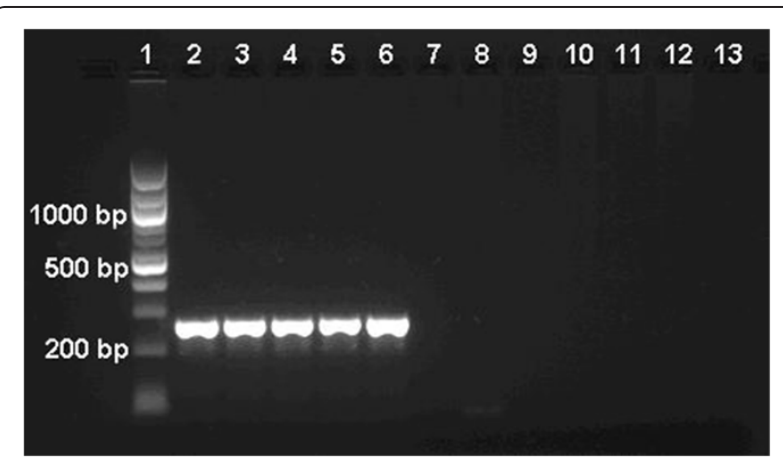

Figure 2 No cross-binding reactions applying new Plasmodium vivax forward primer in modified species-specific 18S PCR [26]. The original Plasmodium vivax primer from a multiplex PCR [26] cross-bonded with Plasmodium falciparum when applied in the modified species-specific 18S PCR [26]. The new Plasmodium vivax (Pv) primer was cross tested against different Plasmodium falciparum (Pf) patient samples with diverse levels of high parasitaemia. The results showed on a $2 \%$ agarose gel. Lane $1=100$ bp DNA Ladder, lanes 2- $6=$ Five different positive $\mathrm{P} \vee$ patient samples, lane $7=2 \% \mathrm{Pf}$, lane $8=2-3 \% \mathrm{Pf}$, lane $9=7 \% \mathrm{Pf}$, lane $10=7-10 \% \mathrm{Pf}$, lane $11=10$ $15 \% \mathrm{Pf}$, lane $12=20 \% \mathrm{Pf}$, and lane $13=$ No template. The product size of $\mathrm{Pv}$ is $241 \mathrm{bp}$.
$P$. malariae in a double infection of $P$. falciparum and $P$. malariae. Microscopy incorrectly evaluated two single infections as double infections with P. falciparum, and incorrectly evaluated a $P$. vivax as $P$. knowlesi infection (Table 4).

\section{Discussion}

The two Plasmodium genus-specific single-round amplification PCR assays presented in this study were as follows: a new mitochondrial PCR employing primers PgMt19 F3\&B3 [21], and a modified 18S PCR employing primers rPLU 6\&5 [9]. By designing single-round amplification assays, the cost-efficiency, turnaround time, contamination risk, and the possibility of technical errors are considerably reduced compared with the nested PCR reference method [10]. Previously published $18 \mathrm{~S}$ primers, rPLU3\&4 [10], were also considered for singleround amplification because of short product size (250 bp versus $1200 \mathrm{bp}$ ), but discarded due to high annealing temperatures, 71 and $75^{\circ} \mathrm{C}$ (contra 63 and $64^{\circ} \mathrm{C}$ for rPLU6\&5). Snounou and Singh $[9,10]$ reported that the $18 \mathrm{~S}$ primers showed reduced sensitivity in single-round PCR amplifications, however this study shows that single-round amplifications have the same sensitivity and specificity as nested PCR given suitable parameters and optimisation. The new PCR programs were designed with a high number of cycles and high concentration of additional magnesium chloride, so that the sensitivity of nested PCR was maintained. Furthermore, due to the quick cycles in the designed programs and the high annealing temperatures, also the specificity of nested PCR was maintained despite the high number of cycles. The results from the dilution series showed that both the single-round amplification assays detected a higher number of parallels with positive DNA at the threshold dilution level of $0.5 \mathrm{p} / \mu \mathrm{l}$ compared to the nested PCR reference method. In the patient material the two single-round assays correctly identified all the positives and negatives found by the nested PCR, with the exception of one sample positive only by mitochondrial PCR and microscopy. Statistically the sensitivity of the mitochondrial PCR was non-inferior to that of the reference nested 18S PCR in this patient cohort as well as in the dilution series (at the threshold $0.5 \mathrm{p} / \mu \mathrm{l}$ dilution). Although the sensitivity of the modified 18S PCR could not be proven to be statistically non-inferior to nested PCR in this limited sample size, the test was 


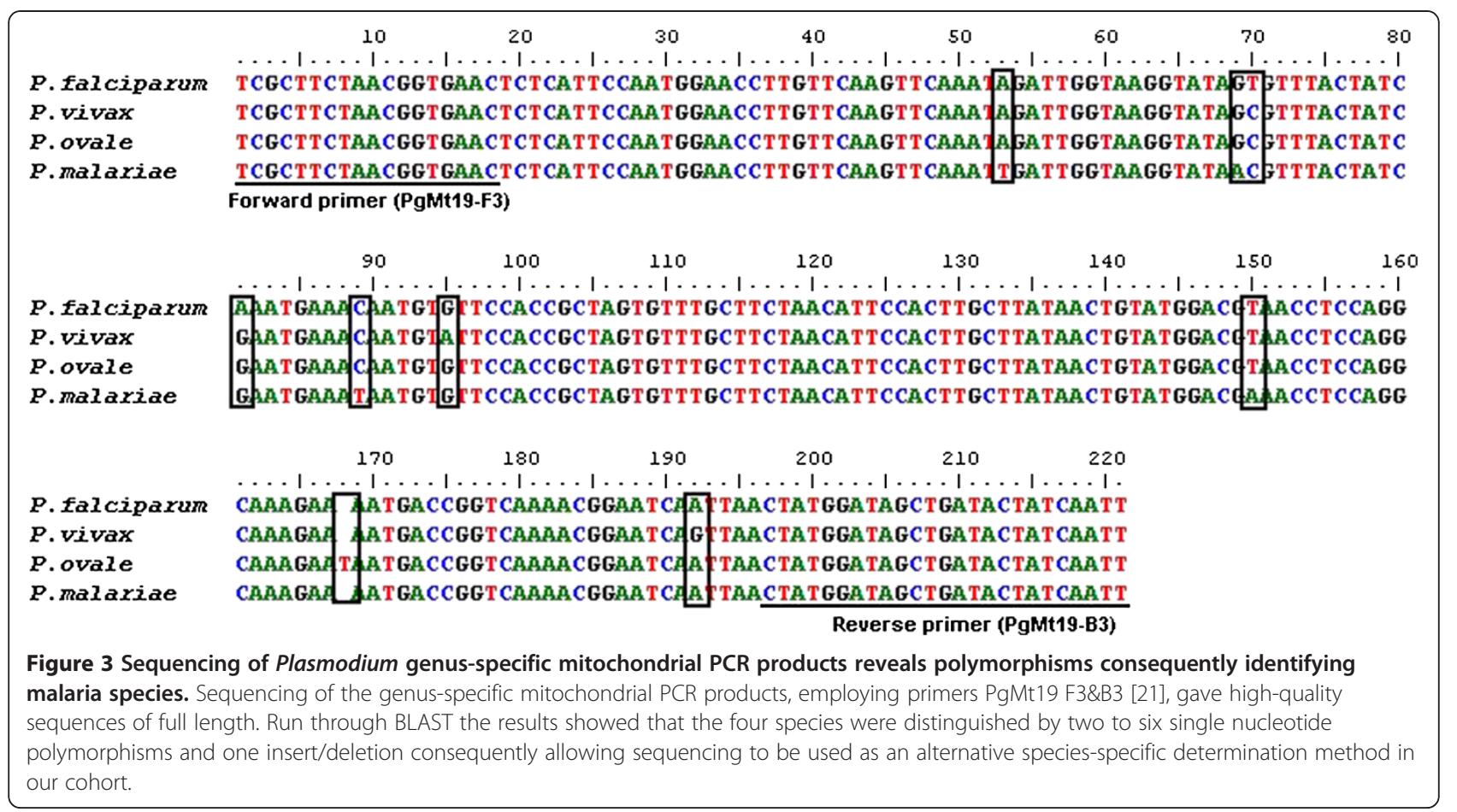

equal to or better than the nested PCR in absolute numbers. The specificities of both single-round amplification assays were statistically non-inferior to that of the nested PCR.

In Plasmodium parasites the mitochondrial genome is presented in a higher copy number than $18 \mathrm{~S}$, especially in gametocytes. While the copy number of $18 \mathrm{~S}$ locus varies depending on strains, and not on the stages of the parasite since it is located on the chromosomal genome

Table 3 Species-specific results among malaria genus positive samples

\begin{tabular}{|c|c|c|c|c|}
\hline $\begin{array}{l}\text { Samples } \\
(n=28)\end{array}$ & $\begin{array}{l}\text { Sequencing } \\
\text { PgMt19 F3 \& B3 [21] }\end{array}$ & $\begin{array}{l}\text { Modified } \\
\text { species- } \\
\text { specific } \\
18 S \text { PCR } \\
{[26]}\end{array}$ & $\begin{array}{l}\text { Species- } \\
\text { specific } \\
\text { nested } \\
18 S \text { PCR } \\
{[10]}\end{array}$ & Microscopy \\
\hline 18 & Pf & Pf & Pf & Pf \\
\hline 1 & Pf & $P f+P m$ & $P f+P m$ & Pf \\
\hline 1 & Pf & Negative & Negative & Pf \\
\hline 3 & $P \vee$ & Pv & $P \vee$ & $P \vee$ \\
\hline 1 & $P \vee$ & PV & $P \vee$ & Negative \\
\hline 1 & $P \vee$ & Pv & $P \vee$ & $P f+P V$ \\
\hline 1 & PV & $P \vee^{1}$ & $P v^{1}$ & $\mathrm{Pk}^{2}$ \\
\hline 1 & Po & Po & Po & $P f+P o$ \\
\hline 1 & $\mathrm{Pm}$ & $\mathrm{Pm}$ & $\mathrm{Pm}$ & Negative \\
\hline
\end{tabular}

Abbreviations: Pf, Plasmodium falciparum; Pv, Plasmodium vivax; Po, Plasmodium ovale; Pm, Plasmodium malariae; Pk, Plasmodium knowlesi. ${ }_{1}^{1}$ Plasmodium knowlesi primers were not included in the assay.

${ }^{2}$ Evaluated as inconclusive species by microscopy, but with emphasis on possible Plasmodium knowlesi infection.
[20], the multiple mitochondrial genome is located in the mitochondrial organelles which can varies in number through the different parasite's stages $[22,23]$. The results from the dilution series, containing ring stage parasites exclusively, showed the same threshold detection level for all three genus-specific PCR assays, though the mitochondrial PCR had highest sensitivity on this detection level. In ring stage parasites the copy number of the mitochondrial genome is approximately three to four times higher than the copy number of the $18 \mathrm{~S}$ locus. Compared to $18 \mathrm{~S}$ PCR, the mitochondrial PCR detected one more sample among the patient material, and notably none of the four $18 \mathrm{~S}$ PCR assays (two genus-/ and two species-specific) detected this sample. The patient had recrudescence following primary $P$. falciparum infection two weeks earlier. Maturation of $P$. falciparum gametocytes takes eight to 10 days $[27,28]$. Although microscopy detected one single ring stage parasite, the difference in sensitivity in this sample is possibly due to submicroscopic gametocytaemia; the copy number of the mitochondrial genome in a gametocyte is approximately 10 to 32 times higher than the copy number of the $18 \mathrm{~S}$ locus.

Due to short product size, $220 \mathrm{bp}$, the amplification time with the new mitochondrial PCR programme takes one-and-a-half hours less than the modified 18S PCR programme, and three hours less than nested 18S PCR [10]. Another advantage of the short product size, was that the mitochondrial PCR products could be easily full-length sequenced. As an alternative to speciesspecific PCR, the SNPs found in the sequences allowed 
Table 4 Seven samples (six patients) with discordant results between the different methods

\begin{tabular}{|c|c|c|c|c|c|c|c|}
\hline Patient & Microscopy & $\begin{array}{l}\text { Parasitaemia by } \\
\text { microscopy }\end{array}$ & $\begin{array}{l}18 \mathrm{~S} \text { PCR } \\
\text { assays }\end{array}$ & $\begin{array}{l}\text { Mitochondrial } \\
\text { PCR/sequencing }\end{array}$ & $\begin{array}{l}\text { Primary/ } \\
\text { recurrent } \\
\text { infection }\end{array}$ & $\begin{array}{l}\text { Place of } \\
\text { infection }\end{array}$ & Interpretation \\
\hline$\overline{P 1}$ & Negative & $0^{1}$ & $\mathrm{Pm}$ & $\mathrm{Pm}$ & Primary & Ghana & Pm overlooked by microscopy \\
\hline P 2 & Negative $^{2}$ & $0^{1}$ & Pv & Pv & Primary & $\begin{array}{l}\text { New } \\
\text { Guinea }\end{array}$ & Pv overlooked by microscopy \\
\hline P 2 & $\mathrm{Pf}, \mathrm{P} \vee$ or $\mathrm{Pk}$ & $1 \%$ & $\mathrm{Pv}$ & $\mathrm{PV}$ & $\begin{array}{l}\text { Recurrent } \\
\text { (Relapse) }\end{array}$ & $\begin{array}{l}\text { New } \\
\text { Guinea }\end{array}$ & $\begin{array}{l}\text { Inconclusive/ incorrect diagnosis by } \\
\text { microscopy }\end{array}$ \\
\hline P 3 & $\mathrm{Pf}+\mathrm{Po}$ & $<1 \%$ & Po & Po & $\begin{array}{l}\text { Recurrent } \\
\text { (Relapse) }\end{array}$ & Uganda & $\begin{array}{l}\text { Over-diagnosed mixed infection by } \\
\text { microscopy }\end{array}$ \\
\hline P 4 & Pf & $<1 \%$ & $P f+P m$ & Pf & Primary & Liberia & $\begin{array}{l}\text { Under-diagnosed mixed infection by } \\
\text { microscopy/sequencing }\end{array}$ \\
\hline P 5 & $\mathrm{Pf}+\mathrm{Pv}$ & $1 \%$ & $\mathrm{PV}$ & $\mathrm{PV}$ & Primary ${ }^{3}$ & SEA or CA & $\begin{array}{l}\text { Over-diagnosed mixed infection by } \\
\text { microscopy }\end{array}$ \\
\hline P 6 & $\mathrm{Pf}^{4}$ & $<1 \%$ & Negative & Pf & $\begin{array}{l}\text { Recurrent } \\
\text { (Recrudescence) }\end{array}$ & Guinea & $\begin{array}{l}\text { Un-detected low Pf parasitaemia by } \\
18 S \text { PCR }\end{array}$ \\
\hline
\end{tabular}

Abbreviations: Pf, Plasmodium falciparum; Pv, Plasmodium vivax; Po, Plasmodium ovale; Pm, Plasmodium malariae; Pk, Plasmodium knowlesi; SEA, South east Asia; $\mathrm{CA}$, Central America.

${ }^{1}$ Not detected any parasites.

${ }^{2}$ Positive microscopy two days later ( $1 \%$ parasitaemia) and then the patient was diagnosed with severe malaria and treatment initiated.

${ }^{3}$ Not given hypnozoite-eradicating treatment and re-admitted four weeks later with relapse of Plasmodium vivax.

${ }^{4}$ Only one parasite detected.

for specific species determination (Figure 3). However the validity of the SNPs warrants further investigation to evaluate if the SNPs are universal in different strains of the species. All of the 28 sequences correctly identified species compared to the species confirmed by either the species-specific 18S PCR assays or microscopy. However, sequencing only identified $P$. falciparum species in a double infection with $P$. malariae, which was detected by both the species-specific $18 \mathrm{~S}$ PCR assays. This can be explained by $P$. falciparum predominance due to its ability to induce high parasitaemia. Despite missing the less numerous species in mixed infections, sequencing of the mitochondrial PCR products can be useful in species diagnosis. Compared to a species-specific multiplex PCR, the sequencing method avoids unwanted cross-bindings due to similar primers' hybridization sites, difficulties in interpreting results due to similar product sizes, and lower sensitivity due to competing primers. Due to high quality sequences this method only requires one reaction for every positive sample to perform species determination, compared to four/five in non-multiplex species-specific PCR assays. Sequencing of positive mitochondrial PCR products also allows for identification of species where species-specific 18S PCR assays may fail to detect low parasitaemia, as shown in the sample with recrudescence in this patient cohort. The simple and rapid mitochondrial PCR assay, with the advantages of high sensitivity and that species-specific sequencing is possible in positive samples, might therefore have a value in screening purposes, especially in large scale epidemiologic and surveillance studies as well as in diagnostics.

\section{Conclusions}

In this study two simple and rapid single-round amplification assays for detection of malaria were described. The novel mitochondrial PCR was the only method with $100 \%$ sensitivity in this patient cohort, and both its sensitivity and specificity was statistically non-inferior to that of the reference $18 \mathrm{~S}$ nested PCR [10]. The method may be of particular value in samples of low parasitaemia/ gametocytaemia and in large-scale studies. Sequencing of the genus-specific mitochondrial PCR products or developing the assay to real-time PCR could be an alternative to species-specific PCR in species determination.

\section{Competing interests}

The authors declare that they have no competing interests.

\section{Authors' contributions}

CGH designed, developed and optimized the PCR programs, performed the analyses, and wrote the first draft of the manuscript. SCM contributed in the development of the PCR methods. KM contributed in providing the clinical data, and in the clinical interpretations. NL initiated the collection of the patient material. BB performed the statistical analysis. KH was involved in including the reference material. All authors contributed to planning of the study, interpretation of the results, revision of the manuscript, and approved the final version.

\section{Acknowledgements}

This work was funded by Centre for Tropical Infectious Diseases, Haukeland University Hospital, Bergen, Norway.

We want to thank Cecilie Helen Isachsen and Marit Gjerde Tellevik for assistance in the laboratorial work, and Dr. Åse Berg (Stavanger University Hospital, Norway) for providing clinical information. We want to sincerely acknowledge Dr. Michael Libman (McGill University Health Centre, Quebec, Canada) for kindly providing us DNA extracted from patient materials that served as Plasmodium falciparum, Plasmodium vivax, Plasmodium ovale and Plasmodium malariae positive controls and for critical review of the manuscript, and the kind donation of the reference material of $P$. falciparum, 
US 04 F Nigeria XII, from the World Health Organization (WHO) Malaria Specimen Bank, hosted by the Center for Disease Control and Prevention (CDC, Atlanta, USA) with support from the Foundation for Innovative New Diagnostics (FIND)

\section{Author details}

${ }^{1}$ National Centre for Tropical Infectious Diseases, Department of Medicine, Haukeland University Hospital, Bergen, Norway. ${ }^{2}$ Institute of Medicine, University of Bergen, Bergen, Norway.

Received: 21 November 2012 Accepted: 17 January 2013

Published: 22 January 2013

\section{References}

1. Murray CJ, Rosenfeld LC, Lim SS, Andrews KG, Foreman KJ, Haring D, Fullman N, Naghavi M, Lozano R, Lopez AD: Global malaria mortality between 1980 and 2010: a systematic analysis. Lancet 2012, 379:413-431.

2. Milne LM, Kyi MS, Chiodini PL, Warhurst DC: Accuracy of routine laboratory diagnosis of malaria in the United Kingdom. J Clin Pathol 1994, 47:740-742.

3. Guerin PJ, Olliaro P, Nosten F, Druilhe P, Laxminarayan R, Binka F, Kilama WL, Ford N, White NJ: Malaria: current status of control, diagnosis, treatment and a proposed agenda for research and development. Lancet 2002, 2:564-573.

4. Hanscheid T: Current strategies to avoid misdiagnosis of malaria. Clin Microbiol Infect 2003, 9:497-504

5. Ndao M: Diagnosis of parasitic diseases: old and new approaches. Interdisciplin Perspect Infect Dis 2009, 2009:278246.

6. Jaureguiberry G, Hatin I, D'Auriol L, Galibert G: PCR detection of Plasmodium falciparum by oligonucleotide probes. Mol Cell Probes 1990, 4:409-414.

7. Hermsen CC, Telgt DS, Linders EH, van de Locht LA, Eling WM, Mensink EJ, Sauerwein RW: Detection of Plasmodium falciparum malaria parasites in vivo by real-time quantitative PCR. Mol Biochem Parasitol 2001, 118:247-251.

8. Poon LL, Wong BW, Ma EH, Chan KH, Chow LM, Abeyewickreme W, Tangpukdee N, Yuen KY, Guan Y, Looareesuwan S, Peiris JS: Sensitive and inexpensive molecular test for falciparum malaria: detecting Plasmodium falciparum DNA directly from heat-treated blood by loop-mediated isothermal amplification. Clin Chem 2006, 52:303-306.

9. Snounou G, Viriyakosol S, Zhu XP, Jarra W, Pinheiro L, Do Rosario VE, Thaithong S, Brown KN: High sensitivity of detection of human malaria parasites by the use of nested polymerase chain reaction. Mol Biochem Parasitol 1993, 61:315-320.

10. Singh B, Bobogare A, Cox-Singh J, Snounou G, Abdullah MS, Rahman HA: $A$ genus- and species-specific nested polymerase chain reaction malaria detection assay for epidemiologic studies. Am J Trop Med Hyg 1999, 60:687-692.

11. Johnston SP, Pieniazek NJ, Xayavong MV, Slemenda SB, Wilkins PP, da Silva AJ: PCR as a confirmatory technique for laboratory diagnosis of malaria. J Clin Microbiol 2006, 44:1087-1089.

12. Mixson-Hayden T, Lucchi NW, Udhayakumar V: Evaluation of three PCRbased diagnostic assays for detecting mixed Plasmodium infection. BMC Res Notes 2010, 3:88.

13. Poschl B, Waneesorn J, Thekisoe O, Chutipongvivate S, Karanis P: Comparative diagnosis of malaria infections by microscopy, nested PCR, and LAMP in northern Thailand. Am J Trop Med Hyg 2010, 83:56-60.

14. Boonma P, Christensen PR, Suwanarusk R, Price RN, Russell B, Lek-Uthai U: Comparison of three molecular methods for the detection and speciation of Plasmodium vivax and Plasmodium falciparum. Malar J 2007, 6:124.

15. Hanscheid T, Grobusch MP: How useful is PCR in the diagnosis of malaria? Trends Parasitol 2002, 18:395-398.

16. Polley SD, Sutherland CJ, Regan F, Hassan M, Chiodini PL: Increased sensitivity for detecting malaria parasites in human umbilical cord blood using scaled-up DNA preparation. Malar J 2012, 11:62.

17. Berry A, Fabre R, Benoit-Vical F, Cassaing S, Magnaval JF: Contribution of PCR-based methods to diagnosis and management of imported malaria. Med Trop 2005, 65:176-183.

18. Rougemont M, Van Saanen M, Sahli R, Hinrikson HP, Bille J, Jaton K: Detection of four Plasmodium species in blood from humans by $18 \mathrm{~S}$
rRNA gene subunit-based and species-specific real-time PCR assays J Clin Microbiol 2004, 42:5636-5643.

19. Han ET, Watanabe R, Sattabongkot J, Khuntirat B, Sirichaisinthop J, Iriko H, Jin L, Takeo S, Tsuboi T: Detection of four Plasmodium species by genusand species-specific loop-mediated isothermal amplification for clinical diagnosis. J Clin Microbiol 2007, 45:2521-2528.

20. Mercereau-Puijalon O, Barale JC, Bischoff E: Three multigene families in Plasmodium parasites: facts and questions. Int J Parasito/ 2002, 32:1323-1344

21. Polley SD, Mori Y, Watson J, Perkins MD, Gonzalez IJ, Notomi T, Chiodini PL, Sutherland CJ: Mitochondrial DNA targets increase sensitivity of malaria detection using loop-mediated isothermal amplification. J Clin Microbiol 2010, 48:2866-2871.

22. Preiser PR, Wilson RJ, Moore PW, McCready S, Hajibagheri MA, Blight KJ, Strath $\mathrm{M}$, Williamson $\mathrm{DH}$ : Recombination associated with replication of malarial mitochondrial DNA. EMBO J 1996, 15:684-693.

23. Krungkrai J: The multiple roles of the mitochondrion of the malarial parasite. Parasitol 2004, 129:511-524.

24. Berry A, Benoit-Vical F, Fabre R, Cassaing S, Magnaval JF: PCR-based methods to the diagnosis of imported malaria. Parasite 2008, 15:484-488.

25. Ndao M, Bandyayera E, Kokoskin E, Gyorkos TW, MacLean JD, Ward BJ: Comparison of blood smear, antigen detection, and nested-PCR methods for screening refugees from regions where malaria is endemic after a malaria outbreak in Quebec. Canada. J Clin Microbiol 2004, 42:2694-2700

26. Padley D, Moody AH, Chiodini PL, Saldanha J: Use of a rapid, single-round, multiplex PCR to detect malarial parasites and identify the species present. Ann Trop Med Parasitol 2003, 97:131-137.

27. Smalley ME: Plasmodium falciparum gametocytogenesis in vitro. Nature 1976, 264:271-272.

28. Jensen JB: Observations on gametogenesis in Plasmodium falciparum from continuous culture. J Protozool 1979, 26:129-132.

\section{doi:10.1186/1475-2875-12-26}

Cite this article as: Haanshuus et al:: A novel, single-amplification PCR targeting mitochondrial genome highly sensitive and specific in diagnosing malaria among returned travellers in Bergen, Norway. Malaria Journal 2013 12:26

\section{Submit your next manuscript to BioMed Central and take full advantage of:}

- Convenient online submission

- Thorough peer review

- No space constraints or color figure charges

- Immediate publication on acceptance

- Inclusion in PubMed, CAS, Scopus and Google Scholar

- Research which is freely available for redistribution 